\title{
A NOTE ON TWO WEIGHTED DISCRETE CARLEMAN INEQUALITIES
}

\author{
BAOFENG LAI*, RUNQIU WANG AND HAO LIU
}

Abstract. In this paper, we re-examine two weighted discrete Carleman inequalities, discuss their correctness and optimal constants in detail, and get some correct and relatively complete conclusions.

Mathematics subject classification (2020): 26D15.

Keywords and phrases: Weighted, discrete, Carleman inequality, optimal constant.

\section{REFERENCES}

[1] T. CARleman, Sur les fonctions quasi-analytiques, in "Conférences faites au cinquiéme congrés des mathematiciens scandinaves," Helsingfores, 1923, 181-196.

[2] G. H. Hardy, J. E. Littlewood, And G. Pólya, Inequalities, Cambridge Univ. Press, London, 1952.

[3] G. H. HARDy, Notes on some points in the integral calculus, LXIV, Messenger Math. 57 (1928), $12-16$.

[4] L. CARleson, A proof of an inequality of Carleman, Proc. Amer. Math. Soc. 5 (1954), 932-933.

[5] R. RedheFFer, Recurrent inequalities, Proc. London. Math. Soc. 17 (1967), 683-699.

[6] H. AlzeR, A refinement of Carleman's inequality, J. Approx. Theory. 95 (1998), 497-499.

[7] B. YANG, L. DEBnATH, Some inequalities involving the constant e, and an application to Carleman's inequality, J. Math. Anal. Appl. 223 (1998), 347-353.

[8] P. YAN, G. Sun, A strengthened Carleman's inequality, J. Math. Anal. Appl. 240 (1999), 290-293.

[9] X. YAnG, On Carleman's inequality, J. Math. Anal. Appl. 253 (2001), 691-694.

[10] S. Kaijser, L.-E. Persson And A. Öberg, On Carleman and Knopp's inequalities, J. Approx. Theory. 117 (2002), 140-151.

[11] G. Peng, A note on Hardy-type inequalities, Proc. Amer. Math. Soc. 133 (2005), 1977-1984.

[12] C. Mortici, Y. Hu, A Carleman's inequality refinement note, Stud. Res. Ser. Math. Inform. 21 (2011), 41-44.

[13] B. YANG, On Hardy's inequality, J. Math. Anal. Appl. 234 (1999), 717-722.

[14] P. Yan, On the extended Hardy's inequality, Int. J. Math. Math. Sci. 27 (2001), 765-768.

[15] A. ČIŽMEŠIJA, J. PEČARIĆ, On strengthened weighted Carleman's inequality, Bull. Austral. Math. Soc. 68 (2003), 481-490.

[16] C.-P. Chen, W.-S. Cheung, And F. QI, Note on weighted Carleman-type inequality, Int. J. Math. Math. Sci. 3 (2005), 475-481.

[17] G. Sunouchi, N. TAKagi, A generalization of the Carleman's inequality theorem, Proc. Phy-Math. Soc. Japan. 16 (1934), 164-166.

[18] D. S. Mitrinović, J. E. PeČARIĆ, AND A. M. FinK, Inequalities Involving Functions and Their Integrals and Derivatives, Kluwer Academic Publishers, 1991.

[19] J. PeČARIĆ, K. B. StolARsky, Carleman's inequality: history and new generalizations, Aequationes Math. 61 (2001), 49-62. 